\title{
Evaluación intraoperatoria ecocardiográfica durante durante la reparación valvular mitral
}

Iván Iglesias
Carolina Cabrera

Western University, Canadá

Universidad de Valparaíso. Chile

Recibido: 31/08/2020

Aceptado: 15/10/2020

En línea: 31/12/2020

\section{Correspondencia}

Iván Iglesias MD

ecoperioperatorio@gmail.com

Citar como: Iglesias I. Evaluación intraoperatoria ecocardiográfica durante reparo de la válvula mitral Rev Ecocar Pract (RETIC). 2020 (Dic); 3 (3): $52-55$. doi: $10.37615 /$ retic.v3n3a15

Cite this as: Iglesias I. Intraoperative echocardiographic evaluation during mitral valve repair .Rev Ecocar Pract (RETIC). 2020 (Dic); 3 (3): 52-55. doi: 10.37615/retic.v3n3a15.

\section{Palabras clave}

$\triangleright$ Ecocardiografía estructural

$\triangleright$ Examen sistemático

\section{RESUMEN}

La ecocardiografía transesofágica (ETE) se ha establecido como herramienta fundamental durante la cirugía cardiaca. Las principales características de la ETE intraoperatoria incluyen: es estructural, es decir, se enfoca en revisar las estructuras que se van a reparar; sistemática, ya que debe revisarse todo de manera organizada y consistente; funcional, debido a que se determina el impacto funcional de los hallazgos; operacional, es decir, se entiende qué debe hacerse para reparar el problema; responsable, porque los hallazgos se comprueban de inmediato en la sala de operaciones; y comprometida, ya que el resultado de la cirugía debe ser satisfactorio.

\begin{abstract}
Transesophageal echocardiography (TEE) has been established as a basic tool during structural cardiac surgery. The main features of intraoperative TEE include: structural, focused on revising the structures to be repaired; systematic, since everything must be reviewed in an organized and consistent manner; functional, because the functional impact of the findings must be determined; operational, provide the reasons about what must be done to repair the problem; responsible, because the findings are checked immediately in the operating room; and committed, since the result of the surgery must be satisfactory.
\end{abstract}

Examen sistemático de la válvula mitral (VM)

Seguimos la orientación anatómica de Carpentier y examinamos la VM en secuencia sistemática (2) para determinar:

- Mecanismo de Carpentier de la insuficiencia mitral (IM).

- Anatomía de la lesión.

¿Qué debe hacer el cirujano para reparar la VM?. Debemos enfatizar la importancia de un examen sistemático (2). En centros que disponen de ETE tridimensional (3D), el ecocardiografista entrenado va a encontrar un gran apoyo en esta modalidad de imagen. Los videos 1 a 7 muestran un examen sistemático de la VM.

Factores favorables y desfavorables en el reparo de la válvula mitral

Se han identificado factores favorables (prolapso localizado de valva posterior, valvas no redundantes, valva anterior menor de $35 \mathrm{~mm}$ de longitud, valva posterior menor de $15 \mathrm{~mm}$ de longitud, gradiente medio $<2 \mathrm{mmHg}$, jet de regurgitación mitral único) y factores que hacen más difícil el reparo valvular mitral (prolapso de valva anterior, prolapso bivalvar, valvas redundantes, jet de regurgitación múltiple o complejo, compromiso de las comisuras).

Antes de ir a circulación extracorpórea (CEC) es imperativo evaluar si existe algún gradiente en el trayecto de salida ventricular izquierdo y evaluar la motilidad regional ventricular izquierda y derecha. Es importante además evaluar si existe insuficiencia aórtica mayor a leve por las implicaciones para cardioplegia anterógrada. Los videos 8 y 9 muestran ejemplos de patología de difícil reparo.

\section{Examen de la válvula mitral}

a la salida de circulación extracorpórea (CEC)

Un examen sistemático va a incluir: excluir presencia de aire intracavitario, evaluar volumen intracardiaco, evaluar función global y regional biventricular, evaluar la válvula mitral reparada usando un examen sistemático ${ }^{(2)}$ pero, a diferencia del examen pre CEC, haciendo más énfasis en el uso de Doppler color para buscar insuficiencia mitral residual, graduar la importancia de la misma y el mecanismo de Carpentier ${ }^{(1,3)}$ y decidir con el cirujano si el resultado es satisfactorio (Figura 1 y Video 10).

En la Tabla 1 se pueden ver los componentes que se evalúan 


\section{Estudio por imagen}

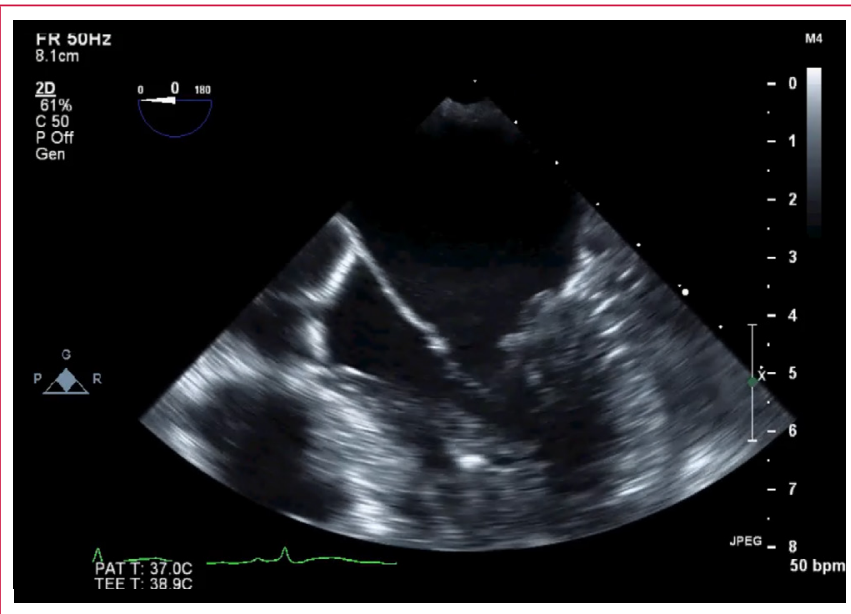

Vídeo 1. Videos 1-7. Secuencia de un examen organizado y completo de la válvula mitral. Video 1. Plano de 5 cámaras. Los 6 segmentos valvares y las 2 comisuras se examinan organizadamente y permiten un diagnóstico preciso de prolapso del festón P2 del velo posterior. La imagen de 3D corrobora este hallazgo

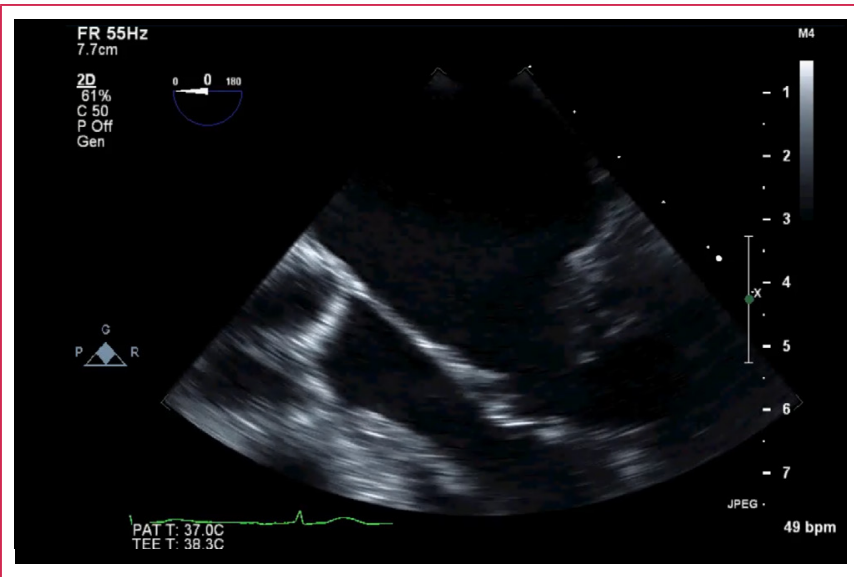

Vídeo 2. Secuencia de un examen organizado y completo de la válvula mitral. Plano de 4 cámaras modificado

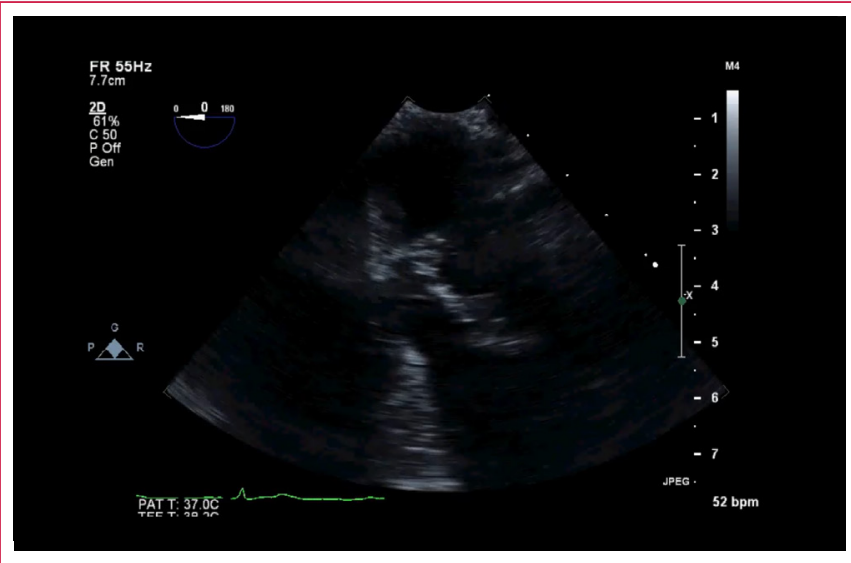

Vídeo 3. Secuencia de un examen organizado y completo de la válvula mitral. Plano de 4 cámaras profundo

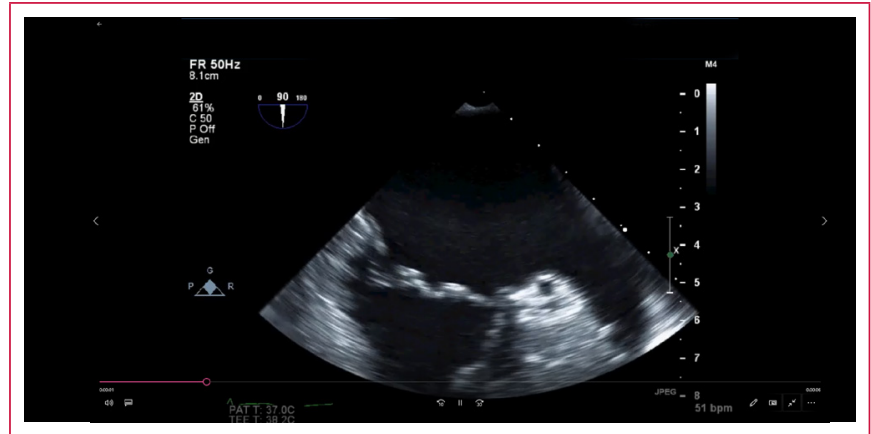

Vídeo 4. Secuencia de un examen organizado y completo de la válvula mitral. Plano de 2 cámaras rotado a la derecha

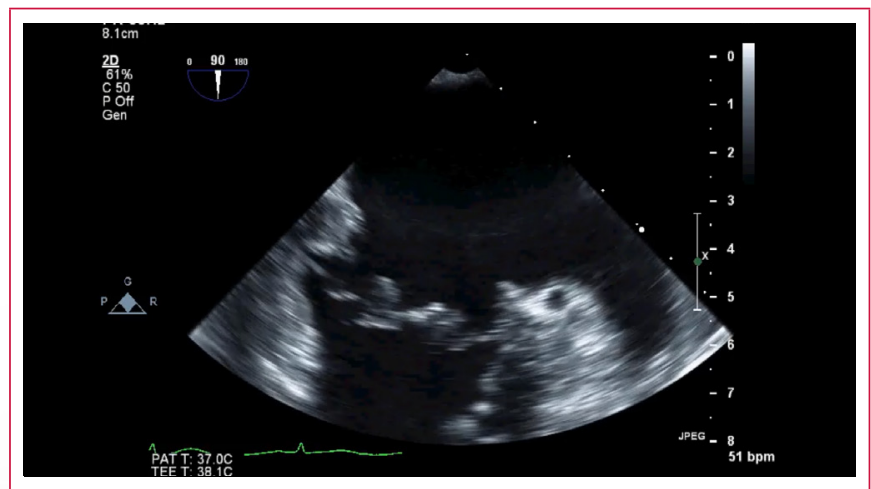

Vídeo 5. Secuencia de un examen organizado y completo de la válvula mitral. Plano comisural

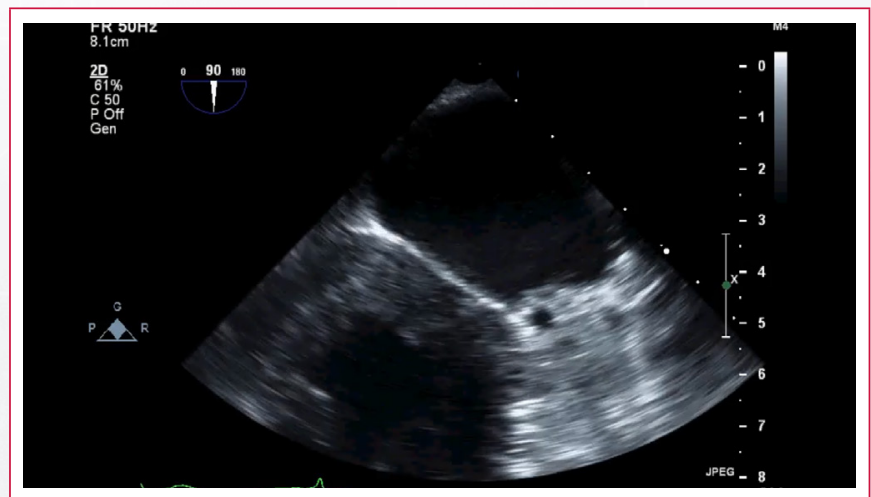

Vídeo 6. Secuencia de un examen organizado y completo de la válvula mitral. Plano de 2 cámaras rotado a la izquierda

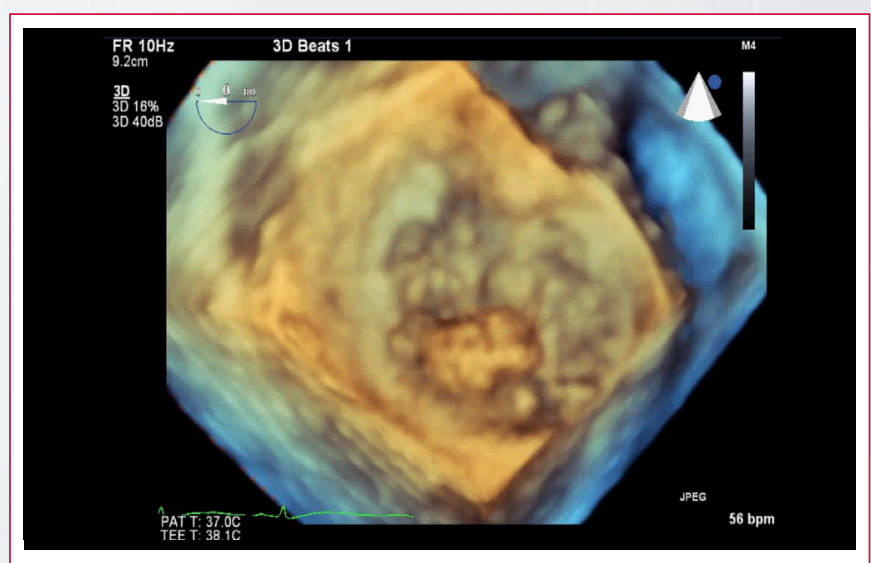

Vídeo 7. Secuencia de un examen organizado y completo de la válvula mitral. Imagen 3D que confirma la lesión del festón P2 del velo posterior. 


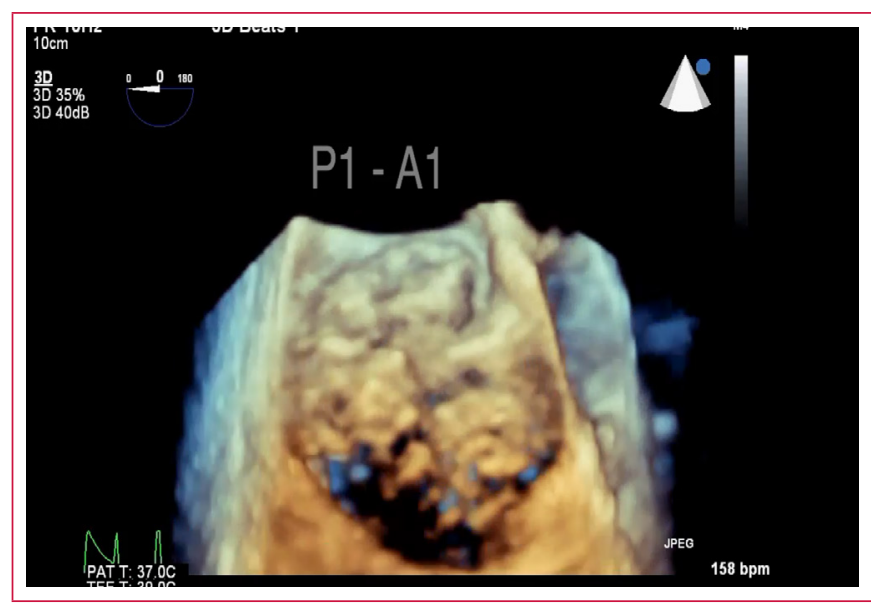

Vídeo 8. Se muestra patología de la comisura anterolateral de la válvula mitral. La patología de valva anterior y comisural es más difícil de reparar

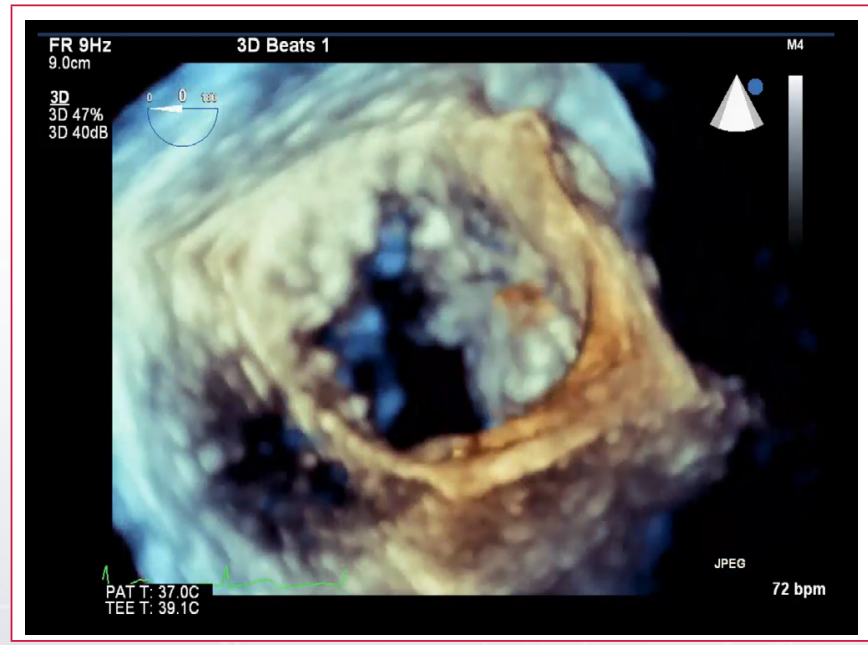

Vídeo 9. Se muestra patología de la valva anterior sobre segmentos A2 y A3 de la válvula mitral

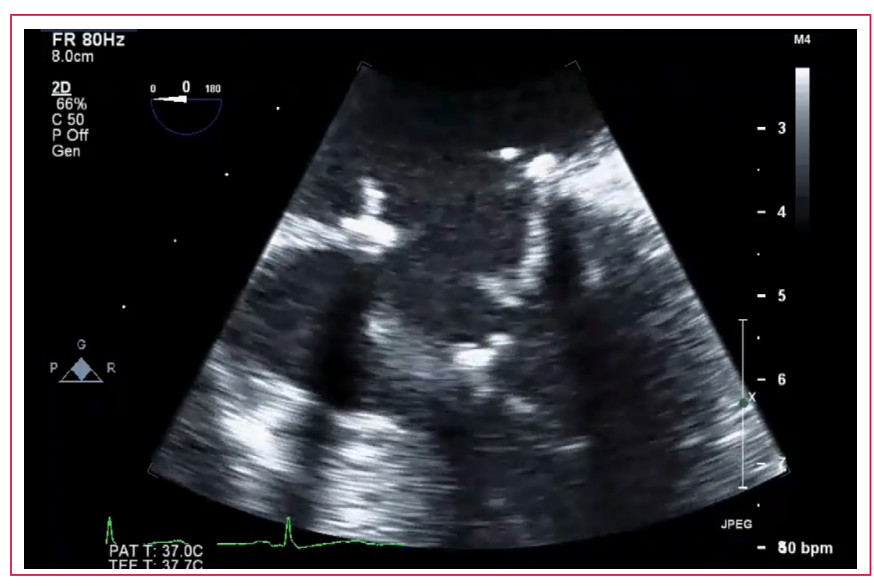

Vídeo 10. Paciente con enfermedad de Barlow, reparo hecho con neocuerdas y anuloplastia. Al intentar salir de bypass se observa SAM con oclusión casi completa del trayecto de salida del VI. Se requirió volver a CEC para corrección quirúrgica con plastia de deslizamiento de valva anterior

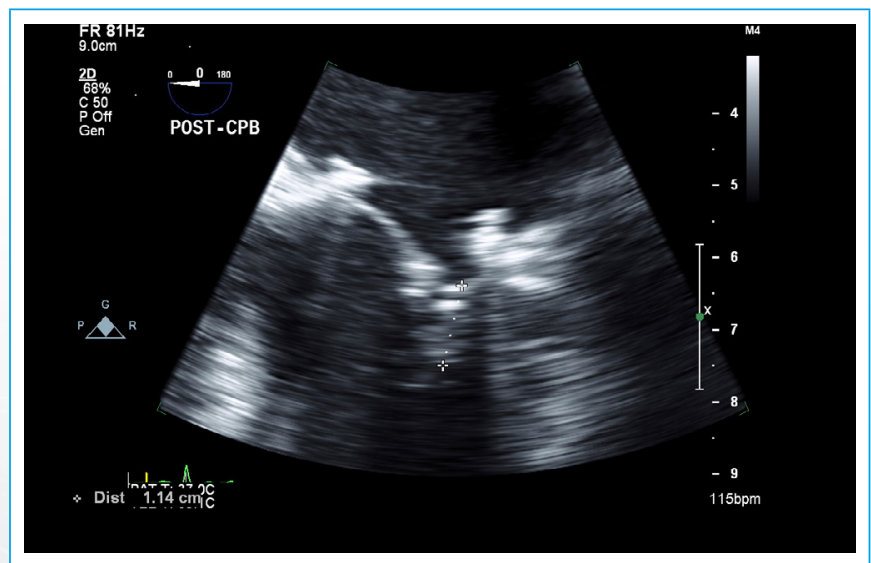

Figura 1. Medición de longitud de coaptación (11.4 mm) post reparo mitral con anuloplastia y neocuerdas

\begin{tabular}{|c|c|c|c|}
\hline & Evaluar & Vista ETE & Meta \\
\hline Saliendo de CEC & No aire intravavitario & 2D esofágica media, eje largo 4 cámaras & No aire antes de retirar vent \\
\hline $\begin{array}{l}\text { Competencia } \\
\text { mitral }\end{array}$ & IM residual & $\begin{array}{l}\text { 2D sistemático con y sin color } \\
\text { 3D auricular con y sin color }\end{array}$ & \begin{tabular}{|l} 
Cero o IM trivial \\
Vena contracta $<3 \mathrm{~mm}$
\end{tabular} \\
\hline \multirow[t]{4}{*}{ Estenosis mitral } & \multirow[t]{4}{*}{ Estenosis iatrogénica } & \begin{tabular}{|l} 
Doppler continuo \\
VM 4 cámaras o eje largo
\end{tabular} & Gradente medio $<5 \mathrm{mmHg}$ \\
\hline & & & Área $>1,8 \mathrm{~cm}^{2}(\mathrm{PHT})$ \\
\hline & & 2D TG eje corto basal & Área $>1,8 \mathrm{~cm}^{2}$ (2D planimetría) \\
\hline & & $3 \mathrm{D}$ vista ventricular & Área $>1,8 \mathrm{~cm}^{2}$ (3D planimetría) \\
\hline $\begin{array}{l}\text { Coaptación } \\
\text { intervalvar }\end{array}$ & Loguitud de coaptación & Eje largo o 4 cámaras & Coaptación $<8$ mm \\
\hline $\begin{array}{l}\text { Trayecto de } \\
\text { salida ventricular }\end{array}$ & SAM & $\begin{array}{l}\text { - Eje largo con y sin color } \\
\text { - TG eje largo doppler continuo }\end{array}$ & $\begin{array}{l}\text { No SAM } \\
\text { No cambio en gradiente pre CEC } \\
\end{array}$ \\
\hline \multirow[t]{2}{*}{$\begin{array}{l}\text { Función } \\
\text { biventricular }\end{array}$} & \multirow[t]{2}{*}{$\begin{array}{l}\text { Presencia de disfunción izquierda } \\
\text { o derecha }\end{array}$} & $\begin{array}{l}\text { Esofágica media } 4 \text { cámaras y tracto entrada- } \\
\text { salida derecho }\end{array}$ & Función VD preservada \\
\hline & & 4, 2 cámaras y eje largo TH eje corto papilar & Función VI preservada, no cambios motilidad regional \\
\hline $\begin{array}{l}\text { Insuficiencia } \\
\text { aórtica }\end{array}$ & Neo insuficiencia & Eje largo color & Cero \\
\hline
\end{tabular}

Tabla 1. Mediciones sugeridas para evaluar reparo valvular mitral post circulación extracorpórea. Tabla original de Iván Iglesias. Abreviaturas: CEC: circulación extracorpórea, IM: insuficiencia mitral, SAM: systolic anterior motion (movimiento anterior sistólico mitral), ETE: ecocardiograma transesofágico, 2D: bidimensional, 3D: tridimensional, VM: válvula mitral, TG: transgástrico, PTH: pressure half time (tiempo de hemipresión), VD: ventrículo derecho, VI: ventrículo izquierdo. 


\section{Ideas para recordar}

- La evaluación ecocardiográfica sistemática de la válvula mitral es perentoria para el éxito de la plastía.
- La ecocardiografía transesofágica permite en tiempo real estimar si el procedimiento fue exitoso

\section{Bibliografía}

1. Fischer G, Anyanwu A, Adams D. Intraoperative classification of mitral valve dysfunction: the role of the anesthesiologist in mitral valve reconstruction. Journal of cardiothoracic and vascular anesthesia. 2009;23(4):531-43.

2. Lambert A, Miller J, Merrick S, Schiller N, Foster E, Muhiudeen-Russell I, et al. Improved evaluation of the location and mechanism of mitral valve regurgitation with a systematic transesophageal echocardiography examination. Anesthesia and analgesia. 1999;88(6):1205-12.

3. Iglesias I. Intraoperative TEE assessment during mitral valve repair for degenerative and ischemic mitral valve regurgitation. Seminars in cardiothoracic and vascular anesthesia. 2007;11(4):301-5. 